\title{
The Role of Tumor Necrosis Factor in Sepsis ${ }^{1}$
}

\author{
Christopher E. Spooner, Norman P. Markowitz, and Louis D. Saravolatz
}

Division of Infectious Diseases and Hospital Epidemiology, Henry Ford Hospital, Detroit, Michigan 48202; and University of Michigan Medical School, Ann Arbor, Michigan 48109

There is an increasing incidence of sepsis among hospitalized patients. Also, high mortality associated with sepsis and septic shock persists despite appropriate antibiotic therapy. Recent investigations have demonstrated that bacterial antigens stimulate a cascade of cellular mediators or cytokine release. In sepsis and septic shock the response of these cytokines often exceeds natural downregulation and leads to multisystem organ failure and even death in an unacceptably high number of patients. Many investigative studies have shown that tumor necrosis factor (TNF) is the prime mediator of the inflammatory response seen in sepsis and septic shock. Sepsis management in the future will include immune modulating therapy directed against the deleterious effects of cytokines, specifically TNF. This article reviews the current problem of sepsis and the evidence to support the role of TNF in sepsis. Also, recent studies employing monoclonal antibodies against TNF as well as considerations for future studies are discussed. (c) 1992 Academic Press, Inc.

\section{INTRODUCTION}

The persistent high mortality and the increasing incidence of sepsis in hospitalized patients provide justification for continued investigative efforts in understanding and managing this most serious infection. Recent studies have demonstrated that a cascade of mediators are stimulated by bacterial antigens (1-7). Furthermore, measurement of these cellular factors or cytokines in animal models and human cases of sepsis supports the hypothesis that tumor necrosis factor (TNF) is a significant mediator of the clinical manifestations of sepsis. A major therapeutic goal that will be an integral part of managing sepsis in the future will be inhibiting the deleterious effects of cytokines, specifically TNF, while maintaining their beneficial effects. This article reviews the current problem of sepsis and the evidence to support the role of TNF as a mediator of sepsis. In addition the results of published studies employing monoclonal antibodies against TNF

\footnotetext{
${ }^{1}$ Presented as part of a symposium entitled "Future Directions of Cytokine and Immunoglobulin Therapy," January 11-12, 1991, Tucson, AZ.
}

are reviewed and considerations for future trials are discussed.

\section{SEPTICEMIA AND THE SEPSIS SYNDROME}

Septicemia is the 13th leading cause of death in the United States. Data from the National Hospital Discharge Survey of the Centers for Disease Control's National Center for Health Statistics have demonstrated a $139 \%$ increase in the rate of septicemia from 1979 to 1987 (8). The rate rose from $73.6 / 100,000$ persons to $175.9 / 100,000$ persons. Although the rate increased among all age groups, the increase was greatest for persons over 65 years of age. In addition, septic shock is the most common cause of death among intensive care unit patients in the United States (5). Mortality rates from septic shock range from 40 to $90 \%$ (9-12). In a survey of septic patients seen at our institution in $1989,35 \%$ died within the first 30 days and $50 \%$ within 180 days. This is consistent with rates seen at other tertiary institutions which are seeing an increase in mortality compared to data from the early 1980s.

Without uniformity of definitions, publications dealing with clinical trials or the epidemiology of sepsis, bacteremia, and septic shock may not be compared. The septic syndrome is an extremely important clinical entity since it serves as an early predictor of the more serious complications associated with septic shock and multiorgan failure. This is especially relevant when designing clinical trials which are aimed at blocking cellular mediators or bacterial toxins released early in the septic process.

The definition of the septic syndrome is based on easily acquired clinical data $(9,13)$. The first criterion is clinical evidence of infection. There is not a requirement for any positive culture. Furthermore, the patient must exhibit the following signs: fever $\left(>101^{\circ} \mathrm{F}\right)$ or hypothermia $\left(<96^{\circ} \mathrm{F}\right)$, tachycardia ( $>90$ beats $/ \mathrm{min}$ ), and tachypnea $(>20$ breaths/min while breathing spontaneously). The patients must also demonstrate at least one of the following manifestations of inadequate organ function or perfusion: altered mental status, hypoxemia $\left(P_{\mathrm{a}} \mathrm{O}_{2}<70\right)$, elevated plasma lactate level, or oliguria $(<30 \mathrm{ml}$ for at least $1 \mathrm{hr})$. Using these criteria, Bone et al. reported on 191 patients of which $45 \%$ 
developed bacteremia and $35 \%$ were in septic shock (9). Twenty-five percent developed the adult respiratory distress syndrome. The overall mortality for the sepsis syndrome was initially reported at $25 \%$; however, recent data suggested that the mortality may be closer to $50 \%$. In an analysis of risk factors, Bone et al. noted a mortality of only $13 \%$ when shock was not present, but this rose to $43 \%$ when shock developed after admission (9). This definition of the septic syndrome allows one to look at the spectrum of this illness and provides a window of opportunity for interventional clinical trials. Early therapy can halt the progression of a disease with a low mortality to one in which the effects of bacterial toxins and cellular mediators have resulted in significant organ damage and an associated high mortality.

\section{PHYSIOLOGIC EFFECTS OF TNF}

Cytokines, produced by activated macrophages, serve to orchestrate a host-defense response against infection, injury, or inflammation. At times this response may lead to chronic inflammation, shock, multisystem organ failure, and even death. One of the most important mediators of the inflammatory response is TNF. Human TNF alone has been shown to be able to conduct the complex orchestration of events leading to the inflammatory response.

Although TNF was first isolated by Carswell et al. in 1975 (14), the discovery was led by the knowledge that infection caused central necrosis of some tumors, especially sarcomas, in both animals and humans. This observation was made by William B. Coley, a New York City surgeon. In the late 19th century, Coley treated 160 patients with killed bacteria, called "Coley's toxins," to treat sarcomas (15). The basis for the use of bacteria in treating tumors came from a number of isolated reports on erysipelas-induced tumor regression (16). Coley's toxins reproduced many of the symptoms of bacterial infection such as fever and chills without causing actual infection. Interest in the potential use of bacteria for the treatment of cancer was to remain alive in the laboratory. In 1943, Shear and his colleagues at the National Cancer Institute isolated and identified the active component of gram-negative bacteria, now called lipopolysaccharide (LPS) (17). These investigators went on to show that LPS could cause hemorrhagic necrosis of tumors, as well as an increase in animals' resistance to new bacterial infections.

In the 1950s Old and Benacerraf isolated and identified the bacterial agent Bacillus Calmette-Guerin, or BCG. BCG was also shown to increase an animal's resistance to subsequent bacterial infection and tumor growth. Carswell et al. went on to show that mice injected with both LPS and BCG produced large amounts of anti-tumor factors which they named "tumor necrosis factor" (14). Old was then able to show that TNF was secreted by macrophages (18).
In 1985, TNF was rediscovered by Cerami and coworkers. In searching for a substance responsible for anorexia and weight loss in chronic disease, these investigators isolated a cytokine synthesized by macrophages. This cytokine appeared responsible for profound wasting and lipemia in rabbits with trypanosomiasis and, therefore, was named "cachectin" (19)

Beutler and Cerami cloned the cDNA for mouse cachectin in 1986 (20) and proved it to be identical in homology to TNF- $\alpha$ cloned 1 year earlier by the researchers at Genentech Inc. (So. San Francisco, CA) (21). TNF- $\beta$ was also cloned by Genentech and found to occur on the same chromosome (chromosome 6 in humans) as TNF- $\alpha$. TNF- $\beta$ has $30 \%$ homology to TNF- $\alpha$ and binds to the same receptor, and for the most part elicits the same response. TNF- $\beta$ is produced primarily by $\mathrm{T}$ lymphocytes and therefore is called "lymphotoxin."

Once TNF was discovered, work was begun to develop a recombinant human tumor necrosis factor (rhTNF), for use as treatment for cancer. In 1987, researchers at the Institute of Cancer Research in London, England, treated 18 patients with advanced cancer with intravenous rhTNF (22). The drug produced febrile reactions at all doses although these were preventable by the administration of indomethacin and steroids. Doses at or above $9 \times 10^{5} \mathrm{u}$ were associated with hypotension, abnormal liver enzymes, leukopenia, and mild renal impairment in a substantial number of patients. These observations supported the biologic importance of TNF as an endogenous pyrogen and mediator of endotoxic shock.

In 1988, researchers at Dana Farber Cancer Institute, in Boston, administered rhTNF to 19 patients using a 5-day continuous infusion. The maximum toler ated dose was associated with undetectable TNF levels $(<0.2 \mathrm{u} / \mathrm{ml})$. Certain tumor cells lines require TNF levels $>1.0 \mathrm{u} / \mathrm{ml}$ for the maximum desired in vitro cytotoxicity (23). Attempts to utilize rhTNF as therapy for cancer have been unsuccessful due to the occurrence of systemic toxicity before therapeutic levels can be reached. Recently, researchers have developed a way to expose only the patient's tumor to TNF, avoiding systemic toxicity, through gene transfer therapy. This type of therapy involves introducing the gene for TNF into the patient's tumor-infiltrating lymphocytes. which selectively traffics to the tumor without exposing the whole system.

In June of 1988, researchers at the Brigham and Women's Hospital measured plasma concentrations of TNF- $\alpha$, interleukin-1- $\beta$ (IL-1- $\beta$ ) and interferon (IFN) together with physiologic and hormonal responses, in 21 healthy men after administration of Escherichia coli endotoxin $(4 \mathrm{mg} / \mathrm{kg})$ and during a control period of saline administration (24). Eight subjects received ibuprofen before receiving endotoxin or saline. TNF was the only cytokine that became elevated in the blood- 
stream of these patients. Also, from 60 to $90 \mathrm{~min}$ after endotoxin administration, the elevation of TNF was associated with all other patient responses. Flu-like symptoms of chills, headaches, myalgias, and nausea were most severe when concentrations of circulating TNF were maximal. Fever and stress-related hormone elaboration persisted for $2 \mathrm{hr}$ or more after TNF was no longer detected in the circulation.

\section{INTERACTIONS, SYNERGY WITH OTHER BRMS} (LPS, IL-1, IFN)

TNF, primarily TNF- $\alpha$, plays a central role in the inflammatory response. TNF is one of many cytokines which are involved in a variety of physiologic responses. Cytokines play a major role in the pathophysiology of many diseases including septic shock. Some have already been found to have therapeutic potential. Strategies for new therapies involve the modification of cytokine levels or receptors through the use of immune modulating agents or gene therapy.

New information and new cytokines are continually being reported. The network of cytokines is as diverse as it is complex. Numerous reports now support the hypothesis that TNF synthesis is under strict regulation by other cytokines and immune modulators. It is now known that interferon- $\gamma$ (IFN- $\gamma$ ), when released by activated $\mathrm{T}$ lymphocytes, is a potent macrophage stimulus that can enhance a number of macrophage activation parameters. An increase in macrophage TNF has been shown to be mediated by IFN- $\gamma$. Interestingly, IFN- $\gamma$ is unable to stimulate release of macrophage-derived TNF by itself, but is capable of augmenting LPS-induced TNF release (25).

In 1988, Kunkel et al. showed that LPS-stimulated macrophages could induce a concomitant increase in monokine synthesis, and cyclo-oxygenase-derived prostaglandin $\mathrm{E}_{2}\left(\mathrm{PGE}_{2}\right)$ may act as a control on TNF production (26). Furthermore, in LPS-induced macrophages, TNF is regulated not only by $\mathrm{PGE}_{2}$ but also by an additional cyclo-oxygenase-derived lipid metabolite prostacyclin $\left(\mathrm{PGI}_{2}\right)$, which can also suppress TNF production. This regulatory effect that $\mathrm{PGE}_{2}$ and $\mathrm{PGI}_{2}$ have on TNF production may play an important role in altering the inflammatory response. However, the regulation of the inflammatory responses may only be local, since systemic $\mathrm{PGE}_{2}$ treatment has no in vivo effects on preformed TNF (27).

In 1989, Essner et al. demonstrated the negative effects that IL-4 had upon TNF (28). It has also been shown that transforming growth factor $\beta$ (TGF- $\beta$ ) suppresses TNF production (29). As little as $10 \mathrm{ng} / \mathrm{ml}$ of TGF- $\beta$ was shown to reduce LPS-dependent TNF production by greater than $50 \%$. TGF- $\beta$ was also shown to inhibit the augmenting effect of IFN- $\gamma$ on TNF production. Interestingly, other mediators do not appear to be affected by TGF- $\beta$.

In January of 1990, Takayama et al. found massively elevated monocyte TNF- $\alpha$ and $\mathrm{PGE}_{2}$ production to be occurring simultaneously in trauma patients with sepsis (30). The high concentration of $\mathrm{PGE}_{2}$ did not appear to downregulate the elevated total monocyte TNF- $\alpha$ levels in these patients. Patients in whom sepsis did not develop had total TNF- $\alpha$ concentrations well below the levels of most patients with septic complications. Patients with extremely high total TNF- $\alpha$ levels during Days 1-3 after injury were reported to have a $100 \%$ mortality. The trauma patients with sepsis showed increased total (cell-associated plus secreted) monocyte TNF- $\alpha$ levels occurring concomitantly with massive elevation in their monocyte $\mathrm{PGE}_{2}$ levels. The peak elevation in total monocyte TNF- $\alpha$ levels of patients correlated with septic episodes as early as 3 days before overt sepsis. These data suggest that trauma-induced production of mediators may be counteracting the downregulatory effect of $\mathrm{PGE}_{2}$ on TNF- $\alpha$ mRNA expression, as well as inducing TNF- $\alpha$ production.

The ability of cytokines to influence the production of other cytokines emphasizes the complexity of interactions that may occur with protein mediator synthesis in the inflammatory or other physiologic responses. The host shows considerable efforts to protect itself from excessive TNF levels. Once the immune system is triggered there is a burst of TNF synthesis followed by decreased production. It is suspected that TNF production may be exaggerated during sepsis or septic shock to too great a degree or too early for regulatory factors to prevent its serious systemic toxicity-potentially leading to end-organ failure or death.

\section{TNF INFUSION STUDIES}

In 1986, Tracey and Beutler showed that shock and tissue injury could be elicited by injecting recombinant human cachectin into rats (31). When administered in quantities similar to those produced endogenously in response to endotoxin, rhTNF was shown to produce hypotension, metabolic acidosis, hemoconcentration, and death within minutes to hours. At necropsy, diffuse pulmonary hemorrhage and inflammation, ischemic and hemorrhagic lesions of the gastrointestinal tract, and acute renal tubular necrosis were found. It appeared that rhTNF alone was capable of inducing the many deleterious effects of endotoxin. This was supported by data published in 1987 by Bauss et al. from Heidelberg, Germany, showing that TNF alone could elicit pathophysiologic effects in mice which were indistinguishable from those produced by endotoxin of LPS (32).

Tracey et al. again showed that lethal shock and stress hormonal responses could be demonstrated when TNF- $\alpha$ was administered by intra-arterial infusion in two groups of beagle dogs at lethal $(100 \mathrm{mg} / \mathrm{kg})$ and sublethal $(10 \mathrm{mg} / \mathrm{kg})$ doses. The infusions produced serum cachectin levels ( 1 to $50 \mathrm{nmol} / \mathrm{liter}$ ) similar to 
those seen after experimental endotoxemia (33). The lethal response to cachectin/TNF was characterized by progressive hypotension, shock, and death within $3 \mathrm{hr}$. Histopathologic findings included acute inflammation of pulmonary interstitium with intravascular thrombosis, hemorrhagic necrosis, adrenal medullary necrosis, and acute renal tubular necrosis. TNF infusion induced a significant increase of plasma catecholamines, cortisol, and glucagon in a dose-response manner (34). This study showed that the infusion of rhTNF, at doses that could be produced in response to bacteremia, stimulates progressive hemodynamic collapse, shock, and death. These observations suggested an important role for cachectin/TNF in the mobilization of host energy stores and the development of shock after infection.

\section{TNF IN CLINICAL SEPSIS}

In February 1987, Waage and Espevik reported an association between TNF in serum and fatal outcome in patients with meningococcal disease. Serum samples were taken on admission from 79 patients with meningococcal meningitis, septicemia, or both, and were examined in a highly sensitive bioassay for TNF. TNF was detected in samples from 10 of 11 patients who died but from only 8 of 68 survivors. All 5 patients with serum TNF levels over $440 \mathrm{u} / \mathrm{ml}$ died (35).

In August 1988, Girardin et al. investigated cytokine levels in the serum of children with severe infectious purpura in order to analyze the relation between serum cytokine levels and clinical and biologic risk factors in those children. The data published provided evidence that serum levels of TNF- $\alpha$, IL-1, and IFN- $\gamma$ correlated with the severity of meningococcemia in children (36).

Many investigators began to look at plasma TNF- $\alpha$ levels and mortality in critically ill septic patients. Researchers from the Department of General Surgery, University of Limburg, The Netherlands, measured plasma TNF levels in 43 septic patients suffering from a broad range of diseases. The data obtained demonstrated that sepsis was accompanied by detectable circulating TNF in $25 \%$ of the cases, and for those patients the mortality was twice that for comparable TNF-negative patients (37). Damas et al., from the University of Leige, Belgium, Studied serum TNF- $\alpha$ levels and IL-1 levels in patients developing sepsis in the intensive care unit. They also reported a correlation between TNF- $\alpha$ levels and sepsis severity as well as mortality. In contrast, IL-1- $\beta$ levels did not correlate with the severity of sepsis or mortality (38). Marks et $a l$, at the University of California, San Francisco, assayed serum samples from 86 patients who were enrolled in a prospective randomized trial of the effects of methylprednisolone in sepitc shock. TNF was detectable in 27 of 74 patients with septic shock and only 1 of 12 patients with shock due to other causes. TNF was detected with equal frequency in patients with shock from gram-negative or from gram-positive sepsis. Furthermore, the patients with detectable TNF had a higher incidence and severity of the adult respiratory distress syndrome and a higher mortality rate than did patients without detectable TNF (39).

\section{TNF MONOCLONAL ANTIBODY STUDIES}

With the availability of monoclonal antibody (MAb) hybridoma technology, the feasibility of blocking the deleterious effects of TNF has been realized.

Several animals have been studied to evaluate the protective role of passive immunization against TNF from the effects of endotoxin. Beutler et al. immunized mice with rabbit antibody against murine TNF and challenged them with lethal amounts of LPS (40). There was a significant reduction of mortality at 7 days in mice passively immunized compared to control animals. The protection was dose-dependent and most effective when administered prior to the injection of endotoxin. Of note, the anti-TNF did not abrogate the pyrogenic effects of LPS.

A recent study by Opal et al. demonstrated the efficacy of antibody against TNF in a neutropenic rat model in protecting animals from lethal infection with Pseudomonas aeruginosa (41). This model was established to mimic the pathophysiology of bacterial infection as it occurs in patients receiving cancer chemotherapy. The role of MAb directed against TNF was assessed alone and in combination with antimicrobial agents. TNF MAb was given at Time 0 and $120 \mathrm{hr}$ while the $P$. aeruginosa challenge was given at 0,48 , and $96 \mathrm{hr}$. Intravenous administration of TNF MAb protected $53.3 \%(8 / 15)$ of animals in contrast to no survivors in untreated control animals $(P<0.005)$. When animals were given ciprofloxacin alone, $67 \%$ survived, while the combination treatment group afforded complete protection $(P<0.05)$. The study demonstrated not only that there was a reduction in mortality with TNF MAb, but also that there was a delay in lethality for up to $72 \mathrm{hr}$ in septic animals. Furthermore, the circulating levels of TNF were significantly lower in the animals receiving anti-TNF than in control animals. This study provides objective in vivo data to support the therapeutic additive protective benefit of combination antimicrobial therapy and anti-TNF therapy.

In an additional set of experiments by Silva et al., a hamster MAb directed against TNF- $\alpha$ was developed and administered to mice as prophylaxis and therapy against $E$. coli (42). There was significant improvement in survival at 3 days if given before and up to 1.5 $\mathrm{hr}$ after bacterial challenge. If given at $2.5 \mathrm{hr}$ after bacterial challenge, TNF MAb improved survival from 12 to $25 \%$; however, this difference was not significant. The protective benefit of TNF MAb was present, but less for infection caused by $P$. aeruginosa than that by 
$E$. coli. Furthermore, no significant difference could be seen in mice treated prophylactically with TNF MAb who were neutropenic and infused with Klebsiella pneumoniae when compared to control animals. These same investigators measured TNF- $\alpha$ levels prior to and at various intervals for up to $5 \mathrm{hr}$ after bacterial challenge. A very rapid rise in serum TNF- $\alpha$ levels to 158 $232 \mathrm{ng} / \mathrm{ml}$ occurs between 50 and $90 \mathrm{~min}$ after bacterial infusion and then drops to levels of $10-12 \mathrm{ng} / \mathrm{ml}$ within $5 \mathrm{hr}$, suggesting a very rapid clearance. Peak levels of TNF- $\alpha$ vary with the amount of LPS injected as well as the animal species studied. An additional observation by Silva $e t a l$. was that the entire physiologic responses of mice treated with anti-TNF- $\alpha$ were identical to those of the control group for up to 6-8 hr after bacterial challenge. These observations are consistent with evidence suggesting that the pathophysiologic effects of LPS are due to mediators in addition to TNF- $\alpha$, such as interleukins and interferons. Another explanation for these observations is that the physiologic responses are centrally mediated, where the transfer of passive antibody would not be expected to be effective since central nervous system levels would be difficult to achieve. The major conclusion drawn from this study is that antiTNF treatment of mice prevented events leading to death. Furthermore, this benefit was not due to enhanced bacterial clearance.

Tracey et al. have studied the role of passive immunization against TNF- $\alpha$ in baboons (43). These studies involved evaluating only anti-TNF in prophylactic experiments. Although a limited number of baboons were evaluated, the authors demonstrated that when antiTNF was given to three baboons $1 \mathrm{hr}$ before challenge with a lethal inoculum of $E$. coli, animals were protected against shock but not multiorgan failure leading to death. However, when anti-TNF was administered $2 \mathrm{hr}$ before bacterial challenge, all animals survived and were protected from shock as well as multiorgan failure.

In a subsequent publication using the original model of Tracey et al., Fong et al. reported on the effect of anti-TNF on other cytokines (44). These authors reported that IL-1- $\beta$ appears slightly later than TNF, being detectable by $2 \mathrm{hr}$ and peaking $3 \mathrm{hr}$ after bacterial infusion. IL-6 appeared even later, at $3 \mathrm{hr}$, and continued to rise throughout $8 \mathrm{hr}$ of the experimental observations. Experimental models have suggested that TNF induces the biosynthesis of IL-1 and IL-6 as secondary mediators. Levels of IL-1- $\beta$ and IL-6 were significantly reduced when anti-TNF was given at both 1 and $2 \mathrm{hr}$ before bacterial challenge. These observations add further weight to the importance of TNF as a major mediator of sepsis.

Hinshaw et al. further evaluated the role of antiTNF in a model of lethal $E$. coli-induced shock (45). In these experiments the investigators infused the lethal dose of $E$. coli over $2 \mathrm{hr}$ and the anti-TNF was initiated
30 min after the onset of the $E$. coli infusion. All animals treated with anti-TNF survived the full 7 days of the experiment until they were sacrificed at Day 7 . Furthermore, multiorgan failure was prevented as well as death with anti-TNF. When animals were not treated with anti-TNF, the mean survival time was 19 hr after injection of $E$. coli.

To date there has been only one study published evaluating anti-TNF in humans. In a Letter to the Editor in Lancet, Exley et al. reported on a Phase 1 study of 14 patients with severe septic shock who were administered CB00006, which is a murine IgG $\kappa$ MAb directed against TNF- $\alpha$ (46). Doses were $0.4,2.0$, and $10 \mathrm{mg} / \mathrm{kg}$. In this study CB00006 was found to be safe and without acute side effects. Human anti-mouse antibodies (HAMA) were demonstrated and could be important if repeated administration is required.

\section{CONSIDERATIONS FOR CLINICAL TRIALS WITH ANTI-TNF}

Future trials with TNF MAb will be a fertile area for investigative efforts in the immunotherapy of sepsis. However, these trials will require careful consideration in their design in order to ensure that the critical issues dealing with efficacy are satisfactorily addressed.

Antimicrobial selection should be standardized in an efficacy trial to determine the real benefit of immunotherapy with agents such as TNF MAb and to exclude the confounding effect that could be seen if agents of varying in vitro antimicrobial activity are selected. Although this standardization seems desirable, the in vivo superiority of individual antimicrobial agents or specific combinations is seldom shown in a statistically significant fashion in comparative efficacy trials. Furthermore, resistance patterns, institutional cost considerations, and individual clinician's subjective preferences make standardization of antimicrobial therapy extremely cumbersome. The advantage of not standardizing antimicrobial therapy when evaluating immunotherapeutic modalities is that the study will reflect how these agents might work in the "real world" by current practice standards.

The definition of sepsis represents a most difficult and important consideration for clinical trials. The definition proposed by Bone et al. is accepted by many clinicians, but does have some limitations (9). Patients often do not fulfill all the criteria at a single point in time. Therefore, it is important for the clinician to recognize the "evolving septic syndrome." The patient must manifest the signs of the septic syndrome over a 12-hr period. Even using these criteria, only 40 to $45 \%$ of patients will have associated bacteremias. Furthermore, patients, especially the elderly, will fail to manifest signs of sepsis in the face of positive blood cul. tures. The major advantage of recognizing the evolving septic syndrome is the ability to recognize an earlier 
stage of sepsis in a patient that will progress to septic shock, multiorgan failure, and death. Thus, in spite of its limitations in sensitivity and specificity, Bone's definition probably offers the best current definition of an early predictor of the more serious sequelae of sepsis.

The ability to recognize the septic patient is essential in defining the optimal timing for immunotherapeutic interventions. The general philosophy of "the earlier the better" is clearly supported by the animal studies discussed above. Trials that adhere strictly to a very narrow time period after signs of sepsis appear are most likely to demonstrate a benefit from an intervention directed against the release of TNF. However, this will leave many questions unanswered. Clinical situations in which patients present after $12 \mathrm{hr}$ from their first signs of sepsis will occur. In addition, some patients will present with an incomplete septic syndrome or a chronic septic appearance. These patients who do not meet the criteria for the septic syndrome may still benefit from anti-TNF therapy but will not be evaluated in the initial trials.

The effect of HAMA will be of importance for murine anti-TNF, especially if the patient has previously received monoclonal preparations such as OKT-3 or the murine monoclonal anti-lipid A product. This consideration is important for clinical trials but is more important for future clinical practice where physicians will be using a variety of MAbs in clinical situations.

A final consideration for a clinical trial and the clinical practice of immunotherapy will be the use of combination agents. The potential for combination monoclonal therapy with anti-lipid $A$ and anti-TNF has merit by inhibiting two distinct and important mediators of sepsis. MAbs may also be considered to block other cytokines such as IL-1 and IL- 6 and may become part of an MAb "cocktail" used in the therapy of sepsis.

Clinical trials evaluating the role of TNF MAb will be performed in the near future. These studies will capitalize on biotechnology and our improved understanding of the mediators of sepsis. Antibiotics have a limited role in the management of the patient with septic shock beyond the lysis of the causative bacteria. Therapy directed against circulating toxins or mediators such as TNF has fueled our interest in using immunotherapy to neutralize the deleterious effects of the mediators of septic shock. It is hoped that TNF MAb will provide a major advance to our therapeutic approach to the septic patient.

\section{REFERENCES}

1. Dinarello, C. A., Cannon J. G., Wolff S. M., et al., Tumor necrosis factor (cachectin) is an endogenous pyrogen and induces production of interleukin 1. J. Exp. Med. 163, 1433-1450, 1986.

2. Michie, H. R., Spriggs, D. R., Manogue, K. R., et al., Tumor necrosis factor and endotoxin induce similar metabolic responses in human beings. Surgery 104, 280-286, 1988.

3. Warren, R. S., Starnes, F., Fabrilove, J. L., Oettgen, H. F., and Brennan, M. F., The acute metabolic effects of tumor necrosis factor in humans. Arch. Surg. 122, 1395-1400, 1987.
4. Michie, H. R., and Wilmore, D. W., Sepsis, signals, and surgical sequelae (a hypothesis). Arch. Surg. 125, 531-536, 1990.

5. Parillo, J. E., Parker, M. M., Natanson, C, Suffredini, A. F. Dunner, R. L., Cunnion, R. E., and Osnibene, F. P., Septic shock in humans. Ann. Intern. Med. 113, 227-242, 1990.

6. Tracey, K. J., Beutler, B., Lowry, S. F., et al., Shock and tissue injury induced by recombinant human cachectin. Science 234 . $470-474,1986$.

7. Bauss, F., Droge, W., and Mannel, D. N., Tumor necrosis factor mediates endotoxic effects in mice. Infect. Immun. 55, 16221625, 1987

8. From the Centers for Disease Control, Increase in National Hospital Discharge Survey rates for septicemia-United States, 1979-1987. JAMA 263, 937-938, 1990.

9. Bone, R. C., Fisher, C. J., Clemmer, T. D., Slotman, G. J., Metz, C. A., and Balk, R. A., Sepsis syndrome: A valid clinical entity. Crit. Care Med. 17, 389-393, 1989.

10. Parker, M. M., and Parrillo, J. E., Septic shock: Hemodynamics and pathogenesis. JAMA 250, 3324, 1983.

11. Kreger, B. E., Craven, D. E., Carling, P. C., et al., Gram negative bacteremia. Reassessment of etiology, epidemiology and ecology in 612 patients. Am. J. Med. 63, 332, 1980.

12. Pollack, M. M., Fields, A. I., and Ruttimann, U. E., Distributions of cardiopulmonary variables in pediatric survivors and nonsur vivors of septic shock. Crit. Care Med. 13, 454, 1985.

13. Balk, R. A., and Bone, R. C., The septic syndrome: Definition and clinical implications. Crit. Care Clin. 5, 1-7, 1989.

14. Carswell, E. A., Old, L. J.. Kassel, R. L., Green, S., Fiong, N., and Williamson, B., An endotoxin-induced serum factor that causes necrosis of tumors. Proc. Natl. Acad. Sci. USA 72, 3666 . 1975.

15. Coley, W. B., The therapeutic value of the mixed toxins of the streptococcus of erysipelas and bacillus prodigiosus in the treatment of inoperable malignant tumors with a report of one hundred sixty cases. Am. J. Med. Sci. 112, 251, 1896.

16. Coley, W. B., Contribution to the knowledge of sarcoma. The treatment of sarcoma by inoculation with erysipelas, with a report of three recent cases. Ann. Surg. 14, 199, 1891

17. Shear, M. J., and Turner, F. C., Reactions of mice with primary subcutaneous tumors to injections of hemorrhage producing bacterial polysaccharide. J. Natl. Cancer Inst. 4, 461, 1943.

18. Old, L. J., Tumor necrosis factor. Sci. Am. 258(5), 9-75, 1988.

19. Beutler, B., Mahoney, J., Le Trang, N., Pekala, P., and Cerami, A., Purification of cachectin, a lipoprotein lipase-suppressing hormone secreted by endotoxin-induced RAW 264.7 cells. J. Exp. Med. 161, 984-995, 1985.

20. Beutler, B., and Cerami, A., Cachectin and tumor necrosis factor as two sides of the same biological coin. Nature $320,584-588$. 1986.

21. Pennica, D., Nedwin, G. E., Hayflick, J. S., et al., Human tumor necrosis factor: Precursor structure, expression and homology to lymphotoxin. Nature 312, 724-729, 1984.

22. Selby, P., Hobbs, S., Viner, $\mathrm{C}$, et al., Tumor necrosis factor in man: Clinical and biological observations. Br. J. Cancer 56, 803$808,1987$.

23. Sherman, M. L., Spriggs, D. R., Arthur, K. A., et al., Recombinant human tumor necrosis factor administered as a five-day continuous infusion in cancer patients: Phase I toxicity and effects on lipid metabolism. J. Clin. Oncol. 6(2), 344-350, 1988.

24. Michie, H. R., Manogue, K. R., Spriggs, D. R., et al, Detection of circulating tumor necrosis factor after endotoxin administration. N. Engl. J. Med. 318, 1481-1486, 1988.

25. Aggarwal, B. B., Characterization of receptors for human tumor necrosis factor and their regulation by gamma-interferon. $\mathrm{Na}$ ture $318,665-667,1985$ 
26. Kunkel, S. L., Spengler, M., May, M. A., Spengler, R., Larrick, J. W., and Remick, D., Prostaglandin $E_{2}$ regulates macrophagederived tumor necrosis factor gene expression. J. Biol. Chem. $263,5380,1988$.

27. Ibid.

28. Essner, R., Rhoades, K., McBride, W. H., Morton, D. L., and Economou, J. S., IL-4 downregulates IL-1 and TNF gene expression in human monocytes. J. Immunol. 142, 3857-3861, 1989.

29. Espevik, T., Figari, I. S., Shalaby, M. R., Lackides, G. A., Lewis, G. D., Shepard, H. M., and Palladino, M. A., Inhibition of cytokine production by cyclosporin $A$ and transforming growth factor-beta. J. Exp. Med. 166, 571-576, 1987.

30. Takayama, T. K., Miller, C., and Szabo, G., Elevated tumor necrosis factor alpha production concomitant to elevated prostaglandin $\mathrm{E}_{2}$ production by trauma patients' monocytes. Arch. Surg. 125, 29-35, 1990.

31. Tracey, K. J., and Beutler, B., Shock and tissue injury induced by recombinant human cachectin. Science 234, 470-473, 1986.

32. Bauss, F., Droge, W., and Mannel, D. N., Tumor necrosis factor mediates endotoxic effects in mice. Infect. Immun. 55, 1622$1625,1987$.

33. Tracey, K. J., Beutler, B., and Cerami, A., Cachectin/tumor necrosis factor induces lethal shock and stress hormone responses in the dog. Surg. Gynecol. Obstet. 164, 415-422, 1987.

34. Ibid.

35. Waage, A., and Espevik, T., Association between tumor necrosis factor in serum and fatal outcome in patients with meningococcal disease. Lancet 319, 355-357, 1987.

36. Girardin, E., Grau, G. E., Dayer, J. M., et al., Tumor necrosis factor and interleukin-1 in the serum of children with severe infectious purpura. N. Engl. J. Med. 319, 397-400, 1988.

37. Debets, J. M., Kampmeijer, R., van der Linden, M. P., et al., Plasma tumor necrosis factor and mortality in critically ill septic patients. Crit. Care Med. 17(6), 489-494, 1989.

38. Damas, P., Reuter, A., Gysen, P., et al., Tumor necrosis factor and interleukin-1 serum levels during severe sepsis in humans. Crit. Care Med. 17(10), 975-978, 1989.

39. Marks, J. D., Marks, C. B., Luce, J. M., et al., Plasma tumor necrosis factor in patients with septic shock: Mortality rate, incidence of adult respiratory distress syndrome. Am. Rev. Respir. Dis. 141, 94-97, 1990.

40. Beutler, B., Milsark, I. W., and Cerami, A. C., Passive immunization against cachectin/tumor necrosis factor protects mice from lethal effect of endotoxin. Science 229, 869-871, 1985.

41. Opal, S. M., Cross, A. S., Kelley, N. M., Sadoff, J. C., Bodmer, M. W., Palardy, J. E., and Victor, G. H., Efficacy of a monoclonal antibody directed against TNF in protecting neutropenic rats from lethal infection with $P$. aeruginosa. J. Infect. Dis. 161, 1148-1152, 1990.

42. Silva, A. T., Bayston, K. F., and Cohen, J., Prophylactic and therapeutic effects of a monoclonal antibody to TNF-alpha in experimental gram-negative shock. J. Infect. Dis. 162, 421-427, 1990.

43. Tracey, K. J., Fong, Y., Hesse, D. G., Manogue, K. R., Lee, A. T., and Kuo, G. C., Anti-cachectin/TNF monoclonal antibodies prevent septic shock during lethal bacteremia. Nature 330, 662$664,1987$.

44. Fong, Y., Tracey, K. J., Moldawer, L. L., Hesse, D. G., Manogue, K. R., and Kenney, J.S., Antibodies to cachectin/TNF reduce interleukin-1-beta and interleukin-6 appearance during lethal bacteremia. J. Exp. Med. 170, 1627-1633, 1989.

45. Hinshaw, L. B., Tekamp-Olson, P., Chang, A. C. K., Lee, P. A., Taylor, F. B., Jr., Murray, C. K., Peer, G. T., Emergon, T. E., Jr., Poassey, R. B., and Juo, G. C., Survival of primates in $L_{100}$ septic shock following therapy with antibody to tumor necrosis factor (TNF-alpha). Circ. Shock 30, 279-292, 1990.

46. Exley, A. R., Cohern, J., Buurman, W., Owen, R., Lumley, J., Hanson, G., Aulakh, J. M., Bodner, M., Riddell, A., Stephens, S. and Perry, M., Monoclonal antibody to TNF in severe septic shock. Lancet 335, 1275-1276, 1990.

Received August 23, 1991; accepted September 23, 1991 